BULl. AUSTRAL. MATH. SOC.

VOL. 33 (1986), 81-87.

\title{
TORSION IN THE ADDITIVE GROUP OF RELATIVELY FREE LIE RINGS
}

\section{VESSELIN DRENSKY}

Let $L=L(X)$ be the free Lie ring of countable rank and let $p$ be prime. Then $L\left(V_{p}\right)=L /\left[\left(L^{\prime}\right)^{p}, L\right]$ is the relatively free ring for the variety of Lie rings $V_{p}=\left[N_{p-1}, E\right]$ and $V_{p}$ is defined by the identity

$$
\left[\left[\left[x_{1}, x_{2}\right], \ldots,\left[x_{2 p-1}, x_{2 p}\right]\right], x_{2 p+1}\right]=0 \text {. }
$$

The purpose of this note is to establish that there exist elements of order $p$ in the additive group of $L\left(V_{p}\right)$. Previously, the existence of $p$-torsion was proved by Kuz'min for $p=2$ only. Similar results were obtained for varieties of groups by Gupta when $p=2$ and by Stöhr when $p=3$.

\section{Introduction}

Let $H\left(x_{1}, \ldots, x_{c}\right)$ be a "multilinear" commutator of length $c$, that is, the commutator brackets are placed in the monomial $x_{1} \ldots x_{c}$ in an arbitrary, but fixed way. Let $V$ be the variety of groups determined by the identity $H\left(x_{1}, \ldots, x_{c}\right)=1$. Denote by $F(V)$ the relatively free group of countable rank in $V$ and let $S_{n}(F(V))$ be the $n$-th element of the lower central series of $F(V)$. In the classical case, when $V=N_{c_{1}} \ldots N_{c_{k}}$ is a polynilpotent variety, the factors $S_{n}(F(V)) / S_{n+1}(F(V))$ are torsionfree abelian groups. It was a surprising result due to Gupta [2], that there are elements of order 2 in the centre of $F\left(V_{2}\right)$, the relatively free group of the centre-by-metabelian variety $V_{2}=\left[A^{2}, E\right]$, defined by

$$
\left[\left[\left[x_{1}, x_{2}\right],\left[x_{3}, x_{4}\right] 1, x_{5}\right]=1 .\right.
$$

Received 2 May 1985.

Copyright clearance Centre, Inc. Serial-fee code: 0004-9727/86 $\$ A 2.00+0.00$. 
Kuz'min [3], applying methods of homological algebra, found 2-torsion in the relatively free ring $L /\left[L^{\prime}, L\right]$ of the centre-by-metabelian variety of Lie rings. Here $L=L(X)$ is the free Lie ring of countable rank. Stöhr [5], using ideas of Kuz'min, proved the existence of 3-torsion in the centre of $F\left(V_{3}\right)$ where $V_{3}=\left[N_{2} A, E\right]$. We refer to [6] for a survey of results in this field.

In this note we investigate the variety of Lie rings $V_{p}=\left[N_{p-1} A, E\right]$, which is defined by the polymomial identity

$$
\left[\left[\left[x_{1}, x_{2}\right], \ldots,\left[x_{2 p-1}, x_{2 p}\right]\right], x_{2 p+1}\right]=0
$$

and $p$ is prime. The purpose is to generalize Kuz'min's result and to prove that there exist elements of order $p$ in the additive group of the relatively free ring

$$
L\left(V_{p}\right)=L /\left[\left(L^{\prime}\right)^{p}, L\right]
$$

The following result is obtained:

THEOREM. Let $p$ be prime. Then there exists a non-zero multilinear element $u\left(x_{1}, \ldots, x_{2 p+1}\right) \in L\left(V_{p}\right)$ such that $p u\left(x_{1}, \ldots, x_{2 p+1}\right)=0$.

\section{Preliminaries}

We denote by $Z$ the ring of integers, by $Q$ the field of rationals and by $Z_{p}$ the field with $p$ elements, where $p$ is a fixed prime integer. We consider the free Lie ring $L=L(X), X=\left\{x_{1}, x_{2}, \ldots\right\}$, canonically embedded in the free associative ring $Z<X>$. All tensor products are over 2 . For a given field $K$, the algebras $L_{K}=K \otimes L(X)$ and $K \otimes Z\langle X\rangle$ are isomorphic to the free Lie $K$-algebra and the free associative $K$-algebra, respectively. For convenience, we identity $a \otimes u \in K \otimes Z\langle X\rangle$ and $a u \in K\langle X\rangle$, where $a \in K, u \in Z\langle X\rangle$. Let 
$f\left(x_{1}, \ldots, x_{c}\right)$ be a multilinear polynomial from $L$ and let $W$ be the verbal ideal generated by $f\left(x_{1}, \ldots, x_{c}\right)$. It is wel1-known that $K \otimes L / W$ is the relatively free algebra of the variety of lie $K$-algebras, defined by the identity $f\left(x_{1}, \ldots, x_{c}\right)=0$. All details concerning varieties of Lie algebras can be found in Bahturin [1]. In addition, all commutators are left-normed:

$$
\left[x_{1}, x_{2}\right]=x_{1} x_{2}-x_{2} x_{1},\left[x_{1}, \ldots, x_{c-1}, x_{c}\right]=\left[\left[x_{1}, \ldots, x_{c-1}\right], x_{c}\right] .
$$

\section{The Proof of the Main Result}

Denote by $P_{n}$ the set of all elements of $L$ multilinear in $x_{1}, \ldots, x_{n}$ and let $V_{p}$ be the variety of Lie rings determined by (1). Then

$$
P_{n}\left(V_{p}\right)=P_{n} /\left(P_{n} \cap\left[\left(L^{\prime}\right)^{p}, L\right]\right)
$$

is the additive group of the multilinear polynomials in the relatively free ring of $V_{p}$.

LEMMA 1. Let the finitely generated additive group $P_{n}\left(V_{p}\right)$ be decomposed into a sum of cyclic groups

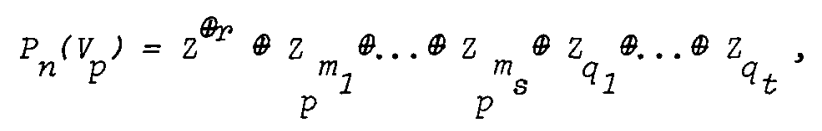

where $p$ does not divide $q_{j}, j=1, \ldots, t$. Then there are elements of order $p$ in $P_{n}\left(V_{p}\right)$ if and only if

$$
\operatorname{dim}_{Q}\left(\left(Q \otimes P_{n}\right) \cap\left[\left(L_{Q}^{\prime}\right)^{p}, L_{Q}\right]\right)>\operatorname{dim}_{Z_{p}}\left(\left(Z_{p} \otimes P_{n}\right) \cap\left[\left(L_{Z}^{\prime}\right)^{p}, L_{Z_{p}}\right]\right) .
$$

Proof. clearly, there is p-torsion in $P_{n}\left(V_{p}\right)$ if and only if $s \neq 0$ in (2), that is when

$$
\operatorname{dim}_{Q} Q \otimes P_{n}\left(V_{p}\right)=r<r+s=\operatorname{dim}_{Z_{p}} Z_{p} \otimes P_{n}\left(V_{p}\right)
$$

For every field $K$,

$$
\operatorname{dim}_{K} K \otimes P_{n}\left(V_{p}\right)=\operatorname{dim}_{K} K \otimes P_{n}-\operatorname{dim}_{K}\left(\left(K \otimes P_{n}^{\prime} n\left[\left(L_{K}^{\prime}\right)^{P}, L_{K}\right]\right) .\right.
$$


This gives the result inmediately since $\operatorname{dim}_{K} K \otimes P_{n}=(n-1) !$.

LEMMA 2. Let

$$
\begin{gathered}
{\left[\left[x_{\sigma(1)}, x_{\sigma(2)}\right] \ldots\left[x_{\sigma(2 p-1)}, x_{\sigma(2 p)}\right], x_{\sigma(2 p+1)}\right],} \\
\sigma(1)<\sigma(2), \sigma(3)<\sigma(4), \ldots, \sigma(2 p-1)<\sigma(2 p), \sigma \in \operatorname{sym}(2 p+1),
\end{gathered}
$$

be all multilinear values in $K<X>$ of

$$
\left[\left[x_{1}, x_{2}\right] \ldots\left[x_{2 p-1}, x_{2 p}\right], x_{2 p+1}\right] \text {, }
$$

$K$ being a field. Then the only linear dependence upon (3) is given by

$$
\left[(\operatorname{sign} \sigma)\left[\left[x_{\sigma(1)}, x_{\sigma(2)}\right] \cdots\left[x_{\sigma(2 p-1)}, x_{\sigma(2 p)}\right], x_{\sigma(2 p+1}\right]=0 .\right.
$$

Proof. It suffices to consider the case $K=Q$ only. By the equality $\left[x_{1}, x_{2}\right]=-\left[x_{2}, x_{1}\right]$, we assume that

$$
\left.\sum a_{\sigma}\left[x_{\sigma(1)}, x_{\sigma(2)}\right] \ldots\left[x_{\sigma(2 p-1)}, x_{\sigma(2 p)}\right], x_{\sigma(2 p+1)}\right]=0 \text {, }
$$

where $a_{\sigma} \in Q$, the sum in (4) is over all $\sigma \in \operatorname{sym}(2 p+1)$ and

$$
a_{\sigma}=-a_{\tau}, \quad \tau=\sigma(2 k-1,2 k), k=1, \ldots, p .
$$

Here the multiplication in the symmetric group is from right to left. We rewrite (4) in the form

$$
\begin{gathered}
\sum_{i=1}^{p}\left[a_{\sigma}\left[x_{\sigma(1)}, x_{\sigma(2)}\right] \ldots\left[x_{\sigma(2 i-1)}, x_{\sigma(2 i)}, x_{\sigma(2 p+1)}\right]\right. \\
\ldots\left[x_{\sigma(2 p-1)}, x_{\sigma(2 p)}\right]=0 .
\end{gathered}
$$

A basis for the vector space spanned by the multilinear products of commutators from $K<X>$ is given in [4]. In particular, the polynomials

$$
\left[x_{j_{1}}, x_{j}\right] \cdots\left[x_{j_{2 i-1}}, x_{j}, x_{j_{2 p+1}}\right] \cdots\left[x_{j_{2 p-1}}, x_{j}\right]
$$

are linearly independent for

$j_{1}<j_{2} ; j_{3}<j_{4} ; \ldots ; j_{2 i-1}<j_{2 i}, j_{2 i-1}<j_{2 p+1} ; \ldots ; j_{2 p-1}<j_{2 p} ; i=1, \ldots, p$.

It follows from (6) that

$$
\begin{aligned}
& \left(a_{\varepsilon}\left[x_{1}, x_{2}, x_{2 p+1}\right]+a_{(1,2,2 p+1}\right)^{\left[x_{2}, x_{2 p+1}, x_{1}\right]} \\
& \left.+a_{(2,2 p+1)}\left[x_{1}, x_{2 p+1}, x_{2}\right]\right)\left[x_{3}, x_{4}\right] \cdots\left[x_{2 p-1}, x_{2 p}\right]=0,
\end{aligned}
$$


where $\varepsilon$ is the identical permutation. The only linear dependence on $\left[x_{1}, x_{2}, x_{2 p+1}\right],\left[x_{2}, x_{2 p+1}, x_{1}\right]$ and $\left[x_{1}, x_{2 p+1}, x_{2}\right]$ is given by the Jacobi identity. Hence

$$
a_{\varepsilon}=a_{(1,2,2 p+1)}=-a_{(2,2 p+1)}
$$

Similarly we obtain the relations

$$
a_{\sigma}=a_{\sigma \rho}, \rho=(2 k-1,2 k, 2 p+1), k=1, \ldots, p .
$$

The permutations $(2 k-1,2 k),(2 k-1,2 k, 2 p+1), k=1, \ldots, p$, generate the symmetric group sym(2p+1). Therefore, we derive from (5) and (7) that $a_{\sigma}=(\operatorname{sign} \sigma) a_{E}$. On the other hand,

$$
\begin{aligned}
& \sum(\operatorname{sign} \sigma)\left[\left[x_{\sigma(1)}, x_{\sigma(2)}\right] \ldots\left[x_{\sigma(2 p-1)}, x_{\sigma(2 p)}\right], x_{\sigma(2 p+1)}\right]
\end{aligned}
$$

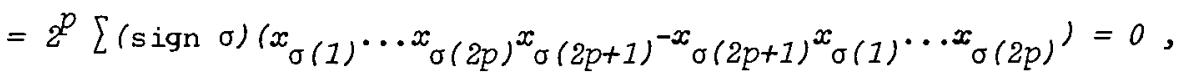

because

$$
\sum(\operatorname{sign} \sigma) x_{\sigma(1)} \cdots_{\sigma(2 p)} x_{\sigma(2 p+1)}=\sum(\operatorname{sign} \sigma) x_{\sigma(2 p+1)} x_{\sigma(1)} \cdots x_{\sigma(2 p)} .
$$

Consequently, the desired linear dependence does exist.

LEMMA 3. The stondard polynomial

$$
\begin{gathered}
S_{2 p}\left(x_{1}, \ldots, x_{2 p}\right)=\sum(\operatorname{sign} \sigma) x_{\sigma(1)}, \ldots x_{\sigma(2 p)} \\
=\sum(\operatorname{sign} \tau)\left[x_{\tau(1)}, x_{\tau(2)}\right] \ldots\left[x_{\tau(2 p-1)}, x_{\tau(2 p)}\right] \in Z_{p}<X>, \\
\sigma, \tau \in \operatorname{Sym}(2 p), \tau(1)<\tau(2), \tau(3)<\tau(4), \ldots, \tau(2 p-1)<\tau(2 p),
\end{gathered}
$$

belongs to $\left(L_{z_{p}^{\prime}}^{\prime}\right)^{p}$.

Proof. Obviously, $S_{2 p}\left(x_{1}, \ldots, x_{2 p}\right)$ is a linear combination of values of the polynomial

$$
h\left(y_{1}, \ldots, y_{p}\right)=\sum y_{\rho(1)} \ldots y_{\rho}(p) \in Z_{p}\langle Y\rangle, \rho \in \operatorname{Sym}(p) .
$$

Hence, it suffices to establish that $h\left(y_{1}, \ldots, y_{p}\right)$ is a Lie element. Having in mind that $Z_{p}<y>$ is a restricted Lie algebra, we obtain that 


$$
\left(y_{1}+\ldots+y_{p}\right)^{p}=y_{1}^{p}+\ldots+y_{p}^{p}+f\left(y_{1}, \ldots, y_{p}\right)
$$

where $f\left(y_{1}, \ldots, y_{p}\right) \in L_{z_{p}}$. The multilinear component of $f\left(y_{1}, \ldots, y_{p}\right)$ is a Lie element as well, and equals $h\left(y_{1}, \ldots, y_{p}\right)$.

Proof of the Theorem. BY [1, Proposition 1, p.115], the relatively free ring of the variety of Lie rings $N_{p-1}$ is torsion-free. Hence

$$
\operatorname{dim}_{K}\left(\left(K \otimes P_{2 p}\right) \cap\left(L_{K}^{\prime}\right)^{p}\right)
$$

does not depend on the choice of the field $K$. Let

$$
u_{s}\left(x_{1}, \ldots, x_{2 p}\right)=\left[\left[x_{s_{1}}, x_{s_{2}}\right], \ldots,\left[x_{s_{2 p-1}}, x_{s_{2 p}}\right], s \in I,\right.
$$

be a basis of $\left(K \otimes P_{2 P}\right) \cap\left(L_{K}^{\prime}\right)^{p}$. Therefore

$$
R_{K}=\left(K \otimes P_{2 p+1}\right) \cap\left[\left(L_{K}^{\prime}\right)^{p}, L_{K}\right]
$$

is spanned by the polynomials

(8) $\left[u_{s}\left(x_{1}, \ldots, x_{i-1}, x_{i+1}, \ldots, x_{2 p+1}\right), x_{i}\right], s \in I, i=1, \ldots, 2 p+1$.

Let us assume first that $K=Q$. Then $S_{2 p}\left(x_{1}, \ldots, x_{2 p}\right)$ is not a lie element in $Q \not>$ and by Lemma 2 the polynomials (8) are linearly independent over $Q$. Now, if $K=2_{p}$, then Lemma 3 gives

$$
S_{2 p}\left(x_{1}, \ldots, x_{2 p}\right)=\sum b_{s} u_{s}\left(x_{1}, \ldots, x_{2 p}\right) \in\left(L_{Z_{p}^{\prime}}^{\prime}\right)^{p} .
$$

In virtue of Lemma 2 we obtain that (8) are linearly dependent over $Z_{p}$ and

$$
\operatorname{dim}_{Z_{p}} Z_{p}<\operatorname{dim}_{Q} R_{Q}
$$

The proof of the theorem follows immediately from Lemma 1 .

\section{References}

[1] J. A. Bahturin, "Lectures on Lie algebras", Studien zur AZgebra cond ihre Anwendungen, 4 (Akademie-Verlag, Berlin, 1978).

[2] C.K. Gupta, "The free centre-by-metabelian groups", J. Austral. Math. Soc. 16 (1973), 294-300. 
[3] J.v. Kuz'min, "Free centre-by-metabelian groups, Lie algebras and D-groups", Izv. Akad. Nauk SSSR, Ser. Mat. 41 (1977), 3-33. Translation: Math. USSR, Izv. 11 (1977), 1-30.

[4] V.N. Latyšev, "Complexity of nonmatrix varieties of associative algebras.I", Algebra Logika, 16 (1977), 149-183. Translation: Algebra Logic, 16 (1978), 98-122.

[5] R. Stöhr, "On free central extensions of free nilpotent-by-abelian groups", Preprint No. 26, Akad. Wiss. DDR, Inst. Math., (1983).

[6] R. Stöhr, "On Gupta representations of central extensions", Math. Z. 187 (1984), 259-267.

Institute of Mathematics

Bulgarian Academy of Sciences

1090 Sofia, P.O. Box 373

Bulgaria 